\title{
Variability of Skin Pharmacokinetic Data: Insights from a Topical Bioequivalence Study Using Dermal Open Flow Microperfusion
}

\author{
Manfred Bodenlenz' • Thomas Augustin ' - Thomas Birngruber' • Katrin I. Tiffner' • \\ Beate Boulgaropoulos ${ }^{1,2}$. Simon Schwingenschuh' - Sam G. Raney ${ }^{3}$. Elena Rantou ${ }^{4}$. \\ Frank Sinner ${ }^{1,2}$ (D)
}

Received: 28 May 2020 / Accepted: 28 August 2020 / Published online: 28 September 2020

(C) The Author(s) 2020

\section{ABSTRACT}

Purpose Dermal open flow microperfusion (dOFM) has previously demonstrated its utility to assess the bioequivalence (BE) of topical drug products in a clinical study. We aimed to characterize the sources of variability in the dermal pharmacokinetic data from that study.

Methods Exploratory statistical analyses were performed with multivariate data from a clinical dOFM-study in 20 healthy adults evaluating the BE, or lack thereof, of Austrian test $(\mathrm{T})$ and U.S. reference $(\mathrm{R})$ acyclovir cream, $5 \%$ products. Results The overall variability of $\log A U C$ values $(\mathrm{CV}: 39 \%$ for $\mathrm{R}$ and $45 \%$ for $\mathrm{T}$ ) was dominated by inter-subject variability (R: 82\%, T: 91\%) which correlated best with the subject's skin conductance. Intra-subject variability was $18 \%(\mathrm{R})$ and $9 \%(\mathrm{~T})$ of the overall variability; skin treatment sites or methodological factors did not significantly contribute to that variability.

Conclusions Inter-subject variability was the major component of overall variability for acyclovir, and treatment site location did not significantly influence intra-subject variability.

Guest Editor: Sam Raney

Frank Sinner

healthca@joanneum.at

HEALTH - Institute for Biomedicine and Health Sciences, Joanneum Research Forschungsgesellschaft m.b.H, Neue Stiftingtalstrasse 2, 8010 Graz, Austria

2 Division of Endocrinology and Diabetology, Department of Internal Medicine, Medical University of Graz, Auenbruggerplatz I 5. 8036 Graz, Austria

3 Division of Therapeutic Performance Office of Research and Standards Office of Generic Drugs, United States (U.S.) Food and Drug Administration, 10903 New Hampshire Avenue, MD 20993 Silver Spring, USA

4 Division of Biostatistics VIII, Office of Biostatistics, Office of Translational Sciences, United States (U.S.) Food and Drug Administration, 10903 New Hampshire Avenue, MD 20993 Silver Spring, USA
These results support a dOFM BE study design with $\mathrm{T}$ and $\mathrm{R}$ products assessed simultaneously on the same subject, where $\mathrm{T}$ and $\mathrm{R}$ treatment sites do not necessarily need to be next to each other. Localized variation in skin microstructure may be primarily responsible for intra-subject variability.

KEY WORDS Topical bioequivalence $\cdot$ inter-and intra-subject variability · dermal open flow microperfusion . microdialysis · acyclovir $\cdot$ skin pharmacokinetics

\begin{tabular}{|c|c|}
\hline \multicolumn{2}{|c|}{ ABBREVIATIONS } \\
\hline ANOVA & analysis of variance \\
\hline$A \cup C$ & $\begin{array}{l}\text { Area under the dermal } \\
\text { concentration-time curve }\end{array}$ \\
\hline $\mathrm{BE}$ & bioequivalence \\
\hline $\mathrm{BMI}$ & body mass index \\
\hline$C_{\max }$ & maximum dermal concentration \\
\hline CV & $\%$ coefficient of variation \\
\hline$d M D$ & dermal microdialysis \\
\hline $\mathrm{dOFM}$ & dermal open flow microperfusion \\
\hline FDA & $\begin{array}{l}\text { United States Food and Drug } \\
\text { Administration }\end{array}$ \\
\hline logAUC values & log-transformed AUC values \\
\hline $\log C_{\max }$ & log-transformed $\mathrm{C}_{\max }$ values \\
\hline PK & pharmacokinetics \\
\hline $\mathrm{R}$ & reference product \\
\hline $\mathrm{T}$ & test product \\
\hline TEWL & transepidermal water loss \\
\hline U.S. & United States \\
\hline
\end{tabular}

\section{INTRODUCTION}

A considerable amount of research has been carried out in recent years to promote new sensitive and discriminating 
methods for the BE assessment of topical dermatological drug products based on pharmacokinetic (PK) endpoints, among them skin stripping (tape stripping), dermal microdialysis (dMD) and dermal open flow microperfusion $(\mathrm{dOFM})$ [1-6]. Although such methods for topical in vivo permeation studies are promising for BE assessments, the resulting data are highly variable - like most of topical PK data. Therefore, the sources of this variability and their potential impact on the outcome of BE assessments remain a subject of major interest.

Characterization of data variability has been performed previously with clinical dMD data in studies on topically applied drugs $[1,7,8]$. Overall variabilities from 40 to $61 \%$ have been observed in vivo $[1,7]$, and differences in skin barrier function between subjects have been assumed to be the major contributor to overall variabilities in topical drug penetration $[1,8]$. Several dMD studies have also investigated intra-subject data variability [1, 9-12], and they have largely attributed this intra-subject variability to biological differences between the individual treatment sites [1, 13]. However, results have certain limitations as they are derived mostly from studies on highly penetrating topical drug products performed with limited probe numbers.

As part of a U.S. Food and Drug Administration (FDA) funded collaborative research effort to evaluate PK-based methods for topical BE assessment, we have recently performed a clinical dOFM study to assess the BE of commercially available acyclovir cream, 5\% products [2]. This study included 20 subjects with six treatment sites per subject and two dOFM probes per treatment site; it delivered a comprehensive data set that verified the topical BE of the reference product $(\mathrm{R})$ to itself and identified the test product $(\mathrm{T})$ as being non-bioequivalent to the $\mathrm{R}$ product. During this study, skin barrier properties were assessed, demographic data recorded, and methodological factors monitored. The resulting data set included data on the dermal PK and on multivariate biological-methodological parameters that might potentially have been associated with the observed variability, thus representing an ideal data set with which to investigate skin PK data variability after topical drug application, and with which to evaluate the sources of that variability.

We therefore aimed to characterize the sources of variability in the dermal PK data using exploratory statistical analyses with this extensive set of data. An understanding of the mechanistic basis for variability in such studies, and the implications for controlling the variability and minimizing the impact of variability on the sensitivity of $\mathrm{BE}$ assessments, is essential in order to optimize dOFM, $\mathrm{dMD}$, and potentially other topical BE study designs, as well as to support reliable power calculations for these clinical BE studies.

\section{MATERIAL AND METHODS}

\section{Clinical dOFM BE study}

The clinical dOFM study included 20 human subjects (Caucasian, age $28 \pm 5$ years, seven female) treated with acyclovir cream, 5\% [2]. In brief, the study design included 6 treatment sites per subject (3 treatment sites on each thigh) and $2 \mathrm{dOFM}$ probes per treatment site. $\mathrm{R}$ and $\mathrm{T}$ were applied in a randomized order of either $\mathrm{R}-\mathrm{R}-\mathrm{T}$ or $\mathrm{T}-\mathrm{R}-\mathrm{R}$ on each thigh. $\mathrm{R}$ was applied twice on each thigh to evaluate the reproducibility of the dOFM data and to serve as positive control for $\mathrm{BE}$ ( $\mathrm{R}$ vs $\mathrm{R}$ ). $\mathrm{T}$ served as negative control and was compared to the $\mathrm{R}$ treatment in the center of the test triad ( $\mathrm{T}$ vs $\mathrm{R}$ ). The dOFM probes were inserted intradermally and probe depth was assessed by longitudinal ultrasound scanning (GE LOGIQe R6 device with linear $22 \mathrm{MHz}$ probe; GE Healthcare, Vienna, Austria) after sampling. Dermal interstitial fluid was continuously sampled with a flow rate of $1 \mu \mathrm{L} / \mathrm{min}$ using sterile perfusate (physiological saline containing $1 \%$ albumin and $600 \mathrm{mg} / \mathrm{dL}$ glucose) from $1 \mathrm{~h}$ pre-dose to $36 \mathrm{~h}$ post-dose, with post-dose sampling intervals of $4 \mathrm{~h}$. Before dosing, the skin temperature of each of the 6 treatment sites was measured (Infrared thermometer TDT8806, Thomson Health Care, France) and transepidermal water loss (TEWL) was measured in duplicate on each thigh (TEWL; Aquaflux AF200; Biox Ltd., London, UK). Skin impedance measurements were performed with a 3-electrode setup in a frequency range from 1 to $100 \mathrm{~Hz}$ [14], and skin conductance at $100 \mathrm{~Hz}$ was used to describe the individual skin barrier property.

Acyclovir cream, 5\% was applied in a homogenous layer $\left(15 \mathrm{mg}\right.$ cream $\left./ \mathrm{cm}^{2}\right)$ to each respective treatment site following a standardized procedure. Thereafter the treatment site was protected by a non-occlusive transparent shield over a duration of $36 \mathrm{~h}$ post-dose [2]. The dose of $15 \mathrm{mg}$ cream/ $\mathrm{cm}^{2}$ took into consideration the low permeation of acyclovir and was selected based on results from pilot studies. Room temperature and relative humidity were tightly controlled throughout the experiment $\left(22 \pm 1{ }^{\circ} \mathrm{C}, 40-60 \%\right.$ relative humidity). Glucose and lactate concentrations in the dOFM samples were measured at the bedside (Super GL; Dr. Müller Gerätebau GmbH, Freital, Germany) as indicators to roughly estimate the stability of the relative recovery, which are straightforward to evaluate at the bedside in each of the 2400 samples. Acyclovir was measured from frozen samples as previously described [2].

\section{Data Set and BE Evaluation Results}

The data set from the clinical dOFM BE study [2] included data on the dermal PK of acyclovir delivered topically from the $\mathrm{R}$ and $\mathrm{T}$ products parameterized as $\mathrm{PK}$ endpoint data 
(area under the dermal concentration-time curve (AUG) and peak/maximum dermal concentration $\left(\mathrm{C}_{\max }\right)$ values), as well as demographic data, data on each subject's skin barrier properties (TEWL, skin conductance), skin temperature, dOFM probe-related data (probe depths), and data derived from methodological monitoring (glucose-loss, recovered lactate and sample mass) (Table 1).

\section{Statistical Analysis}

We performed exploratory statistical analyses of the dOFM data set (Table 1). Data normality was tested with the Kolmogorov-Smirnov test. The overall variability of the dermal endpoint parameter logAUC was expressed as the \% coefficient of variation (CV). Its main components, the interand intra-subject variability, were determined by performing analyses of variance (ANOVAs) with the fixed factors subject, treatment site and probe. We based our analysis on AUC/ $\operatorname{logAUC}$ because this PK parameter incorporates multiple data points, making it not only information-rich and well described by the underlying skin permeation data but also relatively robust, and therefore, most suitable for the identification of the sources of variability. Moreover, and independently, we focused our analysis on the variability of $\mathrm{R}$, as the higher number of replicates for $\mathrm{R}$ facilitated such analysis.

The sources of inter-subject variability were identified using multiple linear regression with a backward elimination technique and the Pearson's product-moment correlation. The following variables were analyzed: sex, age, body mass index (BMI), transepidermal water loss (TEWL), skin conductance, skin temperature (pre-dose), dOFM probe depths after sampling, dOFM sample volumes, and exchange rates of glucose and lactate.

The relevant sources of intra-subject variability were assessed using multiple linear regression and descriptive statistics. To determine whether intra-subject AUC values were normally distributed, we analyzed the distribution within each subject separately, and within the aggregated data for all subjects based on the "normalized" variables $(\mathrm{X}-\mu) / \sigma$, where $\mathrm{X}$ is the original (untransformed) variable, $\mu$ is the intra-subject mean, and $\sigma$ the intra-subject standard deviation: $\mathrm{X}$ is normally distributed within subjects if the "normalized" variable $(\mathrm{X}-\mu) / \sigma$ is normally distributed. Statistics was performed using SAS and plots were created using Origin Pro 2018G.

\section{RESULTS AND DISCUSSION}

\section{Overall variability}

The AUC values showed considerable differences among the 20 subjects and considerable intra-subject variability (Fig. 1, top panels). After log transformation of the AUC values (Fig. 1 , bottom panels), the overall variability $(\mathrm{CV})$ of the dermal endpoint parameter $\log \mathrm{AUC}$ was $39 \%$ for $\mathrm{R}$ and $45 \%$ for $\mathrm{T}$. This is in agreement with the lower range of variabilities observed in previously performed dMD studies (CV ranging from 42 to $93 \%$ ) [11].

An ANOVA of the logAUG values showed that the intersubject variability was the major source of variability (Fig. 2). The remaining 18\% $(\mathrm{R})$ and $9 \%(\mathrm{~T})$ were attributed to intrasubject variability (Fig. 2).

Our ANOVA results showed inter-subject variability to be the greatest source of variability, with a much smaller proportion of the variability arising from intra-subject variability; the intra-subject variability in our study was similarly low or lower compared to the two previously published dMD studies [1, 12]. The dMD study on topical lidocaine products in 8 subjects attributed $61 \%$ of the overall variability to the intersubject-variability and 39\% to intra-subject variability based on an ANOVA analysis [1]. The dMD study on topical ketoprofen in 18 subjects did not calculate the relative contributions of inter- and intra-subject variability based on an ANOVA analysis, but the reported CVs suggest an intersubject variability of approximately $85 \%$ and an intrasubject variability of approximately $15 \%$, i.e. similar to our study [12]. The dominant contribution of inter-subject variability to overall variability has also been reported in studies using tape stripping for the purpose of topical BE assessment [4]. The low intra-subject dOFM variability found in our study indicates a relatively minor influence of any localized variations in skin permeation on overall variability as well as a relatively low contribution of methodological factors to overall variability, which we attribute to the extensive optimization and standardization of the $\mathrm{COFM}$ materials and study procedures. Intra-subject variability includes the factors site and probe, and is the sum of the variations caused by localized variations in skin as well as methodological variations. The ANOVA performed by Benfeldt et al. found equal contributions to variability by the factors site and probe [1]. However, we refrained from discriminating between these two factors because they are interlinked and discrimination by ANOVA might yield misleading results. Pinnagoda et al. performed an ANOVA of TEWL data which indicated that inter-subject differences contributed between approximately $79 \%$ to $92 \%$ (depending on outlier treatment) of the overall variability, while intra-subject differences contributed between approximately $8 \%$ to $21 \%$ of the overall variability [15]. These high inter-subject differences explain, in part, the relatively large numbers of subjects typically required to adequately power comparative clinical endpoint BE studies for topical dermatological drug products.

\section{Inter-Subject variability}

To characterize the skin barrier properties of the subjects, we measured TEWL, skin conductance and skin temperature 
Table I Data set of the acyclovir dOFM BE study

\begin{tabular}{|c|c|c|c|c|c|}
\hline Type of data & Subjects & $\times$ legs & $x$ sites & x probes & $=$ total \\
\hline Subjects demographic data (sex, age, BMI) & 20 & & & & 20 \\
\hline Conductance, TEWL, skin temperature at $\mathrm{t}=0 \mathrm{~h}$ & 20 & 2 & & & 40 \\
\hline Topical treatment sites with drug application $(R, T)$ & 20 & 2 & 3 & & 120 treatment sites \\
\hline dosing $15 \mathrm{mg} / \mathrm{cm}^{2}$ of $\mathrm{R}$ & 20 & 2 & 2 & & 80 treatment sites for $R$ \\
\hline dosing $15 \mathrm{mg} / \mathrm{cm}^{2}$ of $\mathrm{T}$ & 20 & 2 & I & & 40 treatment sites for $T$ \\
\hline dOFM probes inserted in topical treatment sites $(R, T)$ & 20 & 2 & 3 & 2 & 240 probes \\
\hline Probe depths for all dOFM probes at $\mathrm{t}=36 \mathrm{~h}(\mathrm{R}, \mathrm{T})$ & 20 & 2 & 3 & 2 & 240 probe depths \\
\hline dOFM acyclovir profiles, AUCs, $C_{\max }$ for $R$ & 20 & 2 & 2 & 2 & I 60 (| 600 samples) \\
\hline incl. Glucose-loss, volume profiles from -1 to $36 \mathrm{~h}^{\prime}$ & 20 & 2 & 2 & 2 & I 60 (|600 samples) \\
\hline dOFM acyclovir profiles, $A \cup C s, C_{\max }$ for $T$ & 20 & 2 & । & 2 & 80 (800 samples) \\
\hline incl. Glucose-loss, volume profiles from -1 to $36 \mathrm{~h}^{\prime}$ & 20 & 2 & । & 2 & 80 (800 samples) \\
\hline dOFM sampling hours (37 h per probe) ${ }^{\prime}$ & 20 & 2 & 3 & 2 & $8880 \mathrm{~h}$ \\
\hline
\end{tabular}

I $37 \mathrm{~h}$ of sampling: One hour baseline sampling followed by $36 \mathrm{~h}$ of post-dose sampling in $4 \mathrm{~h}$ - intervals ( 10 samples per probe).

The results of the BE evaluation of this study have been published by Bodenlenz et al. [2]. The relative bioavailability of $R$ vs. $R$ and $T$ vs. $R$ has been evaluated based on the conventional BE PK endpoints, AUC and $C_{\max }$ in the dermis, where the criterion for establishing the $B E$ of a $T$ to an $R$ is that the $90 \%$ confidence interval of the geometric mean ratio between the $T$ and $R$ falls within 0.80 and 1.25 . In brief, the positive control products (R vs. R) were accurately and reproducibly confirmed to be bioequivalent [AUCO-36 h $(0.86-1.18)$ and $C_{\max }(0.86-1.2 \mathrm{I})$ ], while the negative control products (T vs. R) were sensitively discriminated not to be bioequivalent for both parameters [AUCO-36 $\mathrm{h}(0.69-1.05)$ and $\left.\mathrm{C}_{\max }(0.61-1.02)\right]$.

(pre-dose). Furthermore, dOFM-related parameters such as sample volumes (flow rate), exchange rates of glucose (loss from perfusate) and exchange rates of lactate (gain from interstitial fluid) were assessed during dOFM sampling. In addition, dOFM probe depths were measured after sampling. Demographic variables (sex, age, BMI) were also analyzed. The parameters were examined in a multiple linear regression analysis with a backward elimination technique. The regression model started with all above mentioned parameters. For the $\mathrm{R}$ product, $39 \%$ of the $\log \mathrm{AUC}$-variation could be explained by two parameters, skin conductance $(p<0.0001)$ and lactate $(p=0.0389)$. For the $\mathrm{T}$ product, $44 \%$ of the $\log \mathrm{AUC}$-variation could be explained by the skin conductance parameter alone $(\mathrm{p}<0.0001)$; the skin conductance parameter was the only parameter consistently associated with logAUC for both, R and T products (Fig. 3).

Pearson's product-moment correlation identified a close relationship between the $\log \mathrm{AUC}$ values and the individual skin conductance $(\mathrm{r}=0.65, p<0.001)$. The relationship between TEWL and logAUG values was weaker $(\mathrm{R}: \mathrm{r}=0.31$, $p=0.054 ; \mathrm{T}: \mathrm{r}=0.37, p=0.017)$. Conductance and TEWL values correlated well with each other for $\mathrm{R}(\mathrm{r}=0.72$, $p<0.0001)$. A possible explanation for the difference in correlation between conductance and TEWL values to the logAUC values might be that results from conductance are more sensitive to the state of hydration of the SC [16]. Increase in hydration of the SC leads to a higher permeability of the SC to topically applied substances [17]. Subjects with a more hydrated SC may therefore have increased AUC values and also increased conductance values. TEWL values may not be elevated to the same extent.
The skin temperature varied from $28.1^{\circ} \mathrm{C}$ to $34.0^{\circ} \mathrm{C}$ between subjects, and also varied slightly between the intrasubject treatment sites. The skin temperature showed a small but statistically significant positive correlation with $\log \mathrm{AUC}$ values $(\mathrm{r}=0.21, p=0.008)$. Skin temperature depends to a certain degree on physiological processes in the viable layers of the skin, such as temperature regulation by skin capillary perfusion (e.g. dilation or constriction) and the rate of local blood flow. Independent of these underlying mechanisms, the skin temperature itself may have the potential to directly affect the rate of drug release from a topically applied formulation, potentially by influencing the drying rate (metamorphosis of the product on the skin) as well as the drug partitioning and/ or diffusion into the skin. However, skin temperature did not significantly differ between subjects and explained only approximately $4 \%$ of the $\log \mathrm{AUC}$-variation and was thus not considered to be a main cause of inter-subject variability in our study.

The mean lactate concentrations in the dOFM samples varied between subjects from 6.7 to $10.4 \mathrm{mg} / \mathrm{dL}$ and showed a small but statistically significant positive correlation to the $\log \mathrm{AUC}$ values for $\mathrm{R}(\mathrm{r}=0.26, p=0.0012)$. For the smaller data set of $\mathrm{T}$ this correlation was not identified. This correlation could be explained by the fact that the lactate concentration in the dOFM sample (at least partially) reflects the relative recovery of molecules from the surrounding of the dOFM probe, which links the recovered lactate to the recovered acyclovir concentration. The correlation of lactate concentrations to the $\log \mathrm{AUC}$ values was statistically significant, but rather low, which indicates a small contribution of the factor relative recovery to the inter-subject variability. Such a correlation 

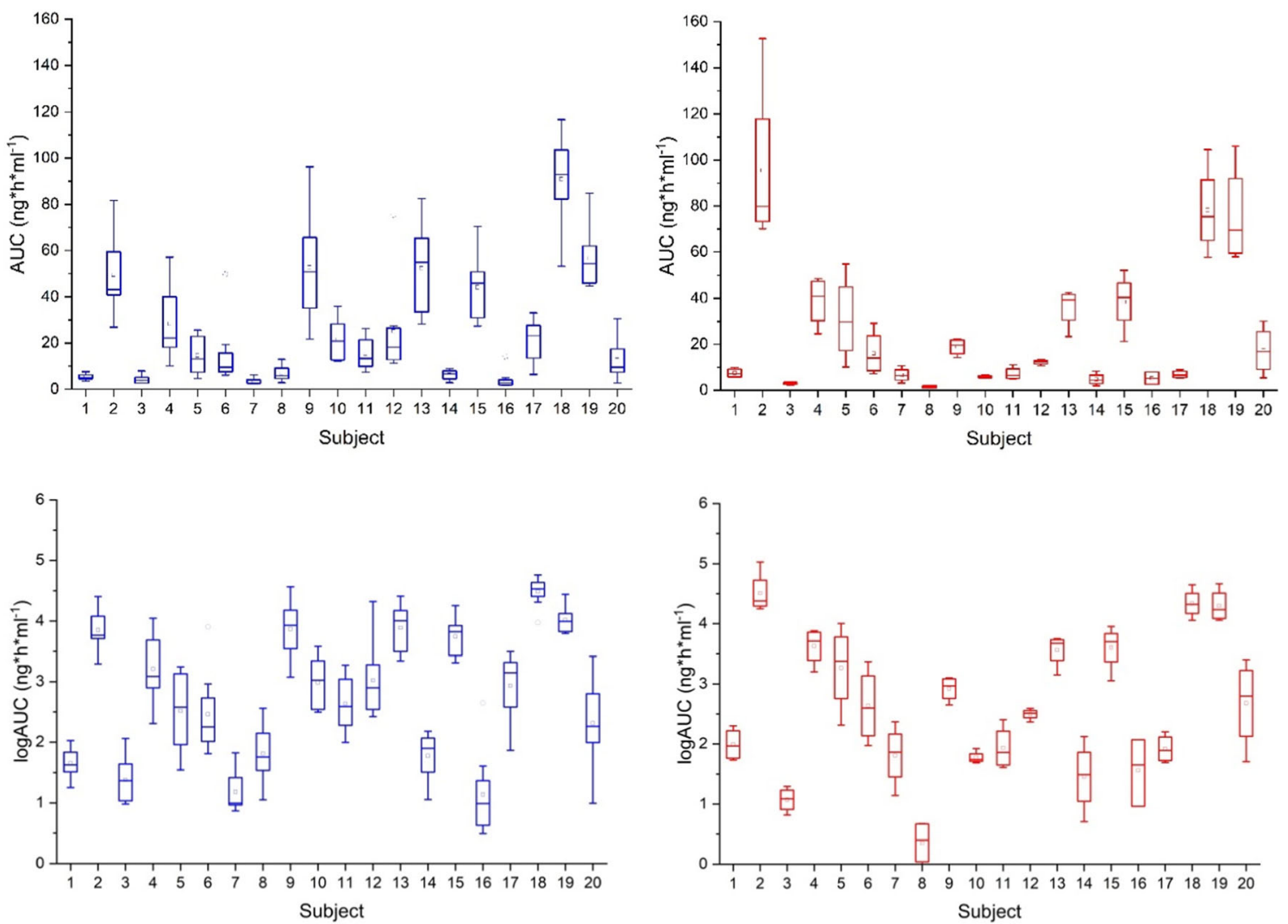

Fig. I AUC values ( $0-36$ h) of all 240 probes in 20 subjects. Top panels: Untransformed AUC values for $\mathrm{R}$ (8 probes per subject, left side) and $\mathrm{T}$ (4 probes per subject, right side). Bottom panels: Log-transformed AUC values for R (left side) and T (right side)

should be explained by the fact that the relative recovery depends on the interstitial fluid content of the tissue, which slightly varies with the individual hydration status of the subject. Lactate is also a descriptor of local metabolism and trauma, which has already been well characterized by others for the use of sampling probes [18]. None of the other dOFM probe-related parameters (probe depth, sample volume, glucose concentration; all $p>0.10)$ showed any correlation with the $\log \mathrm{AUC}$ values.

These results are consistent with results from a lidocaine dMD study that analyzed a number of potential co-variates and observed no correlation between factors like probe depth, room temperature, or humidity and topical drug kinetics [1]. Also Stagni et al. did not find any correlation between the kinetics of dermal drug absorption and probe depth in a dMD study investigating iontophoretically delivered propranolol [13]. However, the apparent lack of a correlation between probe depths and $\log \mathrm{AUC}$ values in our acyclovir dOFM data set is in contrast to results from a dOFM study with the highly lipophilic drug clobetasol-17-propionate, where minor probe depth differences of $0.2 \mathrm{~mm}$ were shown to have an apparent influence on the observed AUC values [19].

\section{Intra-Subject variability}

The AUC values of the $8 \mathrm{R}$ probes showed a positively skewed distribution in 17 of 20 subjects (mean skewness: $0.88 \pm 0.93$, range: -0.82 to 2.50 ). The AUC values normalized based on the individual means (aggregated normalized AUC values) also showed a positively skewed distribution (skewness: + 0.71) (Fig. 4, left side).
Fig. 2 Main sources of variability for $\mathrm{R}$ (left side) and $\mathrm{T}$ (right side) derived from an ANOVA
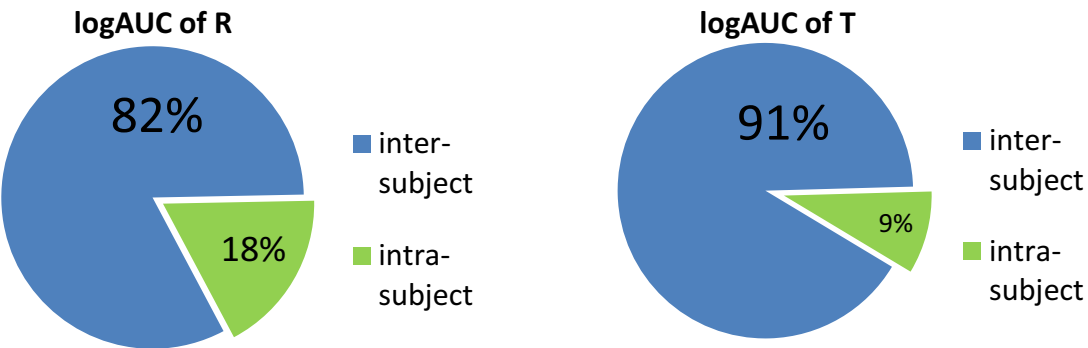

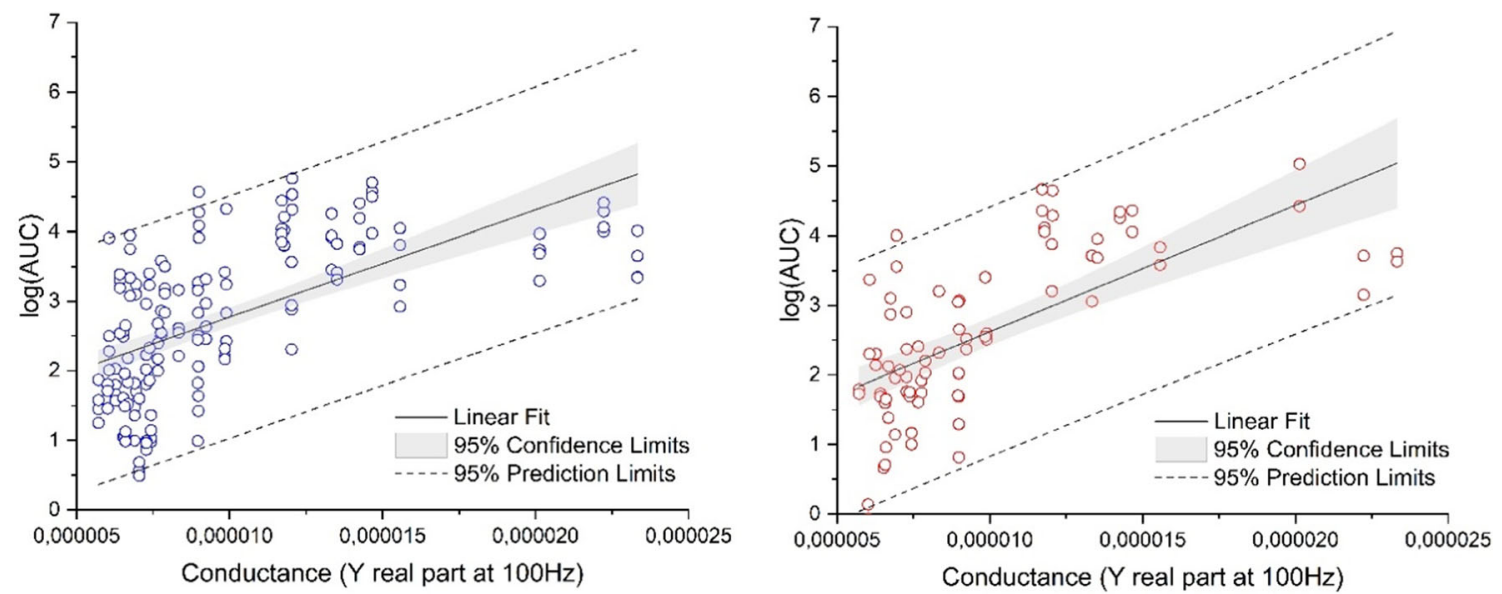

Fig. 3 Multiple linear regression analysis combined with a backward elimination technique identified skin conductance as the sole parameter among those evaluated that appeared to be consistently associated with inter-subject variability. Left side: Relationship of logAUC vs. conductance for R, right side: Relationship of $\log A \cup C$ vs. conductance for $T$

The $\log$ AUC values of the 20 subjects showed a normal distribution (mean skewness: $0.19 \pm 0.87$, range: -1.37 to 1.62). Also, the aggregated normalized logAUC values (Fig. 4 , right side) showed a normal distribution $(p>0.15)$.

No comparable results could be located in the literature from $\mathrm{dMD}$ studies regarding the skewness of the distribution of intra-subject AUC values prior to log-transformation. This might be due to the type of drugs investigated in the dMD studies and/or due to the limited number of $\mathrm{dMD}$ probes typically used per subject.

\section{Factors: site and leg}

We compared the $\mathrm{CV}$ s for the $\log \mathrm{AUC}$ values of the single probes within a treatment site $(45.5 \%)$, on one leg between treatment sites $(49.8 \%)$, and between the legs $(52.4 \%)$; the results indicated that the factor site (and leg) did not significantly contribute to the variability. The subsequent comparison of the logAUC values between the treatment sites on the left and the right leg using regression analysis confirmed good reproducibility between legs for $\mathrm{R}(\mathrm{r}=0.91,2$ vs. 2 treatment sites per subject) and $\mathrm{T}(\mathrm{r}=0.94,1$ vs. 1 treatment site per subject) (Fig. 5).

Previous dMD studies have observed variability between treatment sites on the volar forearm and have attributed them to regional differences in skin barrier function [9] or to possible differences in vasculature between distant treatment sites [12]. However, it is unlikely that local dermal vasculature or local drug clearance significantly differs from site-to-site or from probe-to-probe considering the presence of a dense capillary network of 100 capillaries $/ \mathrm{cm}^{2}$ paralleled by lymphatic vessels in the upper dermis [20]. Benfeldt et al. applied two probes per treatment site and distinguished between the factors site and probe, but did not address potential sources of siterelated variabilities [1]. Our results demonstrated that site and site-related methodological factors did not significantly contribute to intra-subject variability. As a related consideration, skin temperature reductions toward the distal portion of the extremities may contribute slightly to variability based upon our data, which indicated that temperature differences had a small $(4 \%)$ contribution to variability.

\section{Factor: probe}

To assess the contribution of the factor probe to the intrasubject variability, we first calculated the mean $\log \mathrm{AUC}$ value-differences between (i) adjacent probes in the same treatment site $(\Delta 1 \mathrm{~cm})$, (ii) probes in two different treatment sites on the same leg at different distances $(\Delta 3 \mathrm{~cm}$ and $\Delta 4 \mathrm{~cm})$, (iii) probes in different treatment sites on different legs on the same subject ( $\Delta$ leg) (Fig. 6). Most of the intra-subject variability was attributable to variabilities between adjacent probes.

We also investigated the co-factors (probe depth, sample volume, flow rate, recovered lactate and exchange rate of glucose) that could have been attributed to the factor probe. However, analyzing 80 pairs of probes from the treatment sites where $\mathrm{R}$ was applied revealed that none of the probepairs with high differences in AUC values were associated with a deviation in those co-factors. This is consistent with our results from the Pearson's product-moment correlation analysis, which did not identify any significant probe-related cofactors. This is also in agreement with results from a dMD study performed by Kreilgaard et al. showing that data variability was not assignable to the technique itself [9]. Our intrasubject AUC values followed a log-normal distribution, which also did not implicate a methodological origin. Thus, we hypothesized that when using a hydrophilic drug with low permeation, like acyclovir, a significant portion of the observed intra-subject variability might be caused by local skin-related factors, which may influence the skin barrier function, and the 

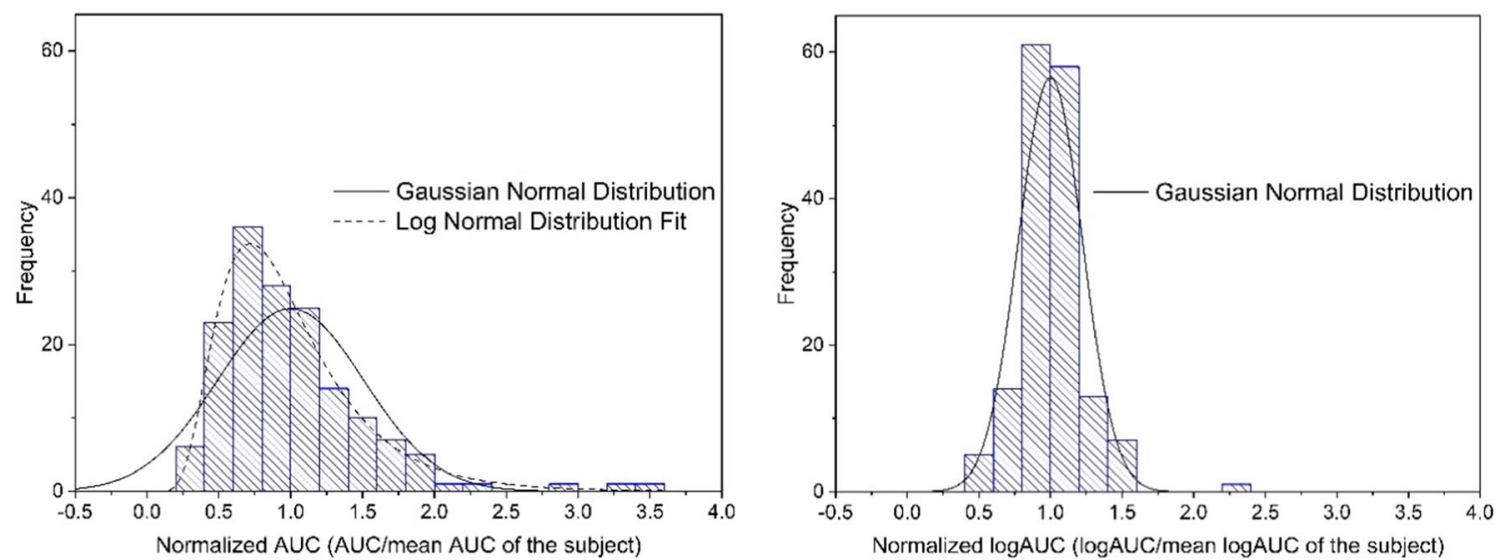

Fig. 4 Distribution of the aggregated normalized intra-subject AUC values for R (0-36 h), Left side: Normalized AUC values. Right side: Normalized logAUC values

drug delivery into the region of skin immediately above the probe.

While there is currently insufficient evidence to support definitive conclusions about the underlying mechanisms by which specific anatomical or structural variations in the skin may influence the rate and extent of topical drug permeation, the influence of skin-related factors on drug permeation has been studied before by In Vitro Permeation Testing (IVPT) [21-25]. Khan et al. have indicated that there is a skewed (not normal) distribution of drug penetration data which occurs when using skin instead of synthetic membranes, which might be caused by skin imperfections such as abrasions, defects or hair follicles [21]. In a large retrospective IVPT study investigating the permeability of tritiated water on skin samples, intra-subject variabilities (CVs: 38.3-115.7\%) have been reported to be even higher than inter-subject variabilities (CV: $37.6 \%$ ). Interestingly, the inter-subject data of this large IVPT data set followed a normal distribution. The intrasubject IVPT data, however, followed a skewed (not normal) distribution in most subjects like seen in the dOFM data [24]. Meidan et al. have attributed this behavior to the presence of local skin differences [24]. Other studies have investigated the influence of skin-related factors on drug permeation using skin with different hair follicle density [26, 27] and different in vitro models [28-32]. Results from those studies have demonstrated that hair follicles constitute a rather fast penetration pathway and that penetration via the follicular route may even be a dominant permeation pathway for hydrophilic drugs. Ogiso et al. have investigated the role of follicular penetration for acyclovir and have found a good correlation between the acyclovir flux and the hair follicle density of the skin $(r=0.666$; $P<0.05)$ [26].

Assuming a hair follicle density in human skin of approximately 17 follicles $/ \mathrm{cm}^{2}$ for men and women at the thighs [33, 34], the probability of a dOFM probe (length: $15 \mathrm{~mm}$, diameter: $0.5 \mathrm{~mm}$ ) to detect acyclovir penetrating via the follicular route from at least one follicle is more than $50 \%$. Therefore, particularly with poorly permeating drugs, the use of more than two probes per treatment site (or per product) might be beneficial as it could further reduce variability and facilitate successful BE assessments comparing the dermal PK of a drug from $\mathrm{T}$ and $\mathrm{R}$ products in efficient $\mathrm{BE}$ studies with
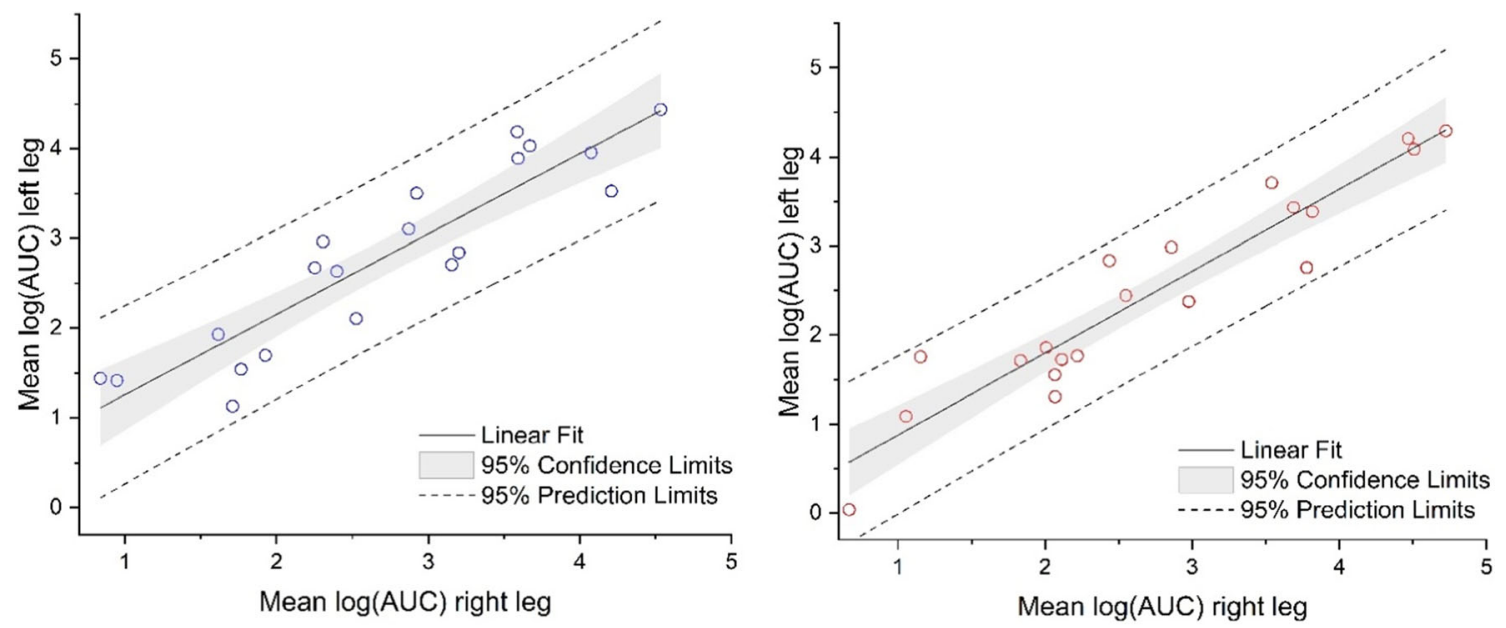

Fig. 5 Comparison of logAUC values between treatment sites on the left and the right leg for $\mathrm{R}$ (left side) and T (right side) 
Fig. 6 Mean differences of IogAUC values between two probes depending on their positions and their distances relative to each other. Upper panel: logAUC values for $\mathrm{R}$ for adjacent probes in the same treatment site $(\Delta \mathrm{I} \mathrm{cm})$ differed by logAUC 0.46 corresponding to an arithmetic mean difference of $59 \%$. The difference between the logAUC values increased only slightly when the two probes were in two different treatment sites $(\Delta 3 \mathrm{~cm}$ and $\Delta 4 \mathrm{~cm}$ ) or at different legs $(\Delta$ leg). Lower panel: $\log A \cup C$ values for $T$ between adjacent probes differed by logAUC 0.44 corresponding to an arithmetic mean difference of $55 \%$. The factor leg did not add any variation
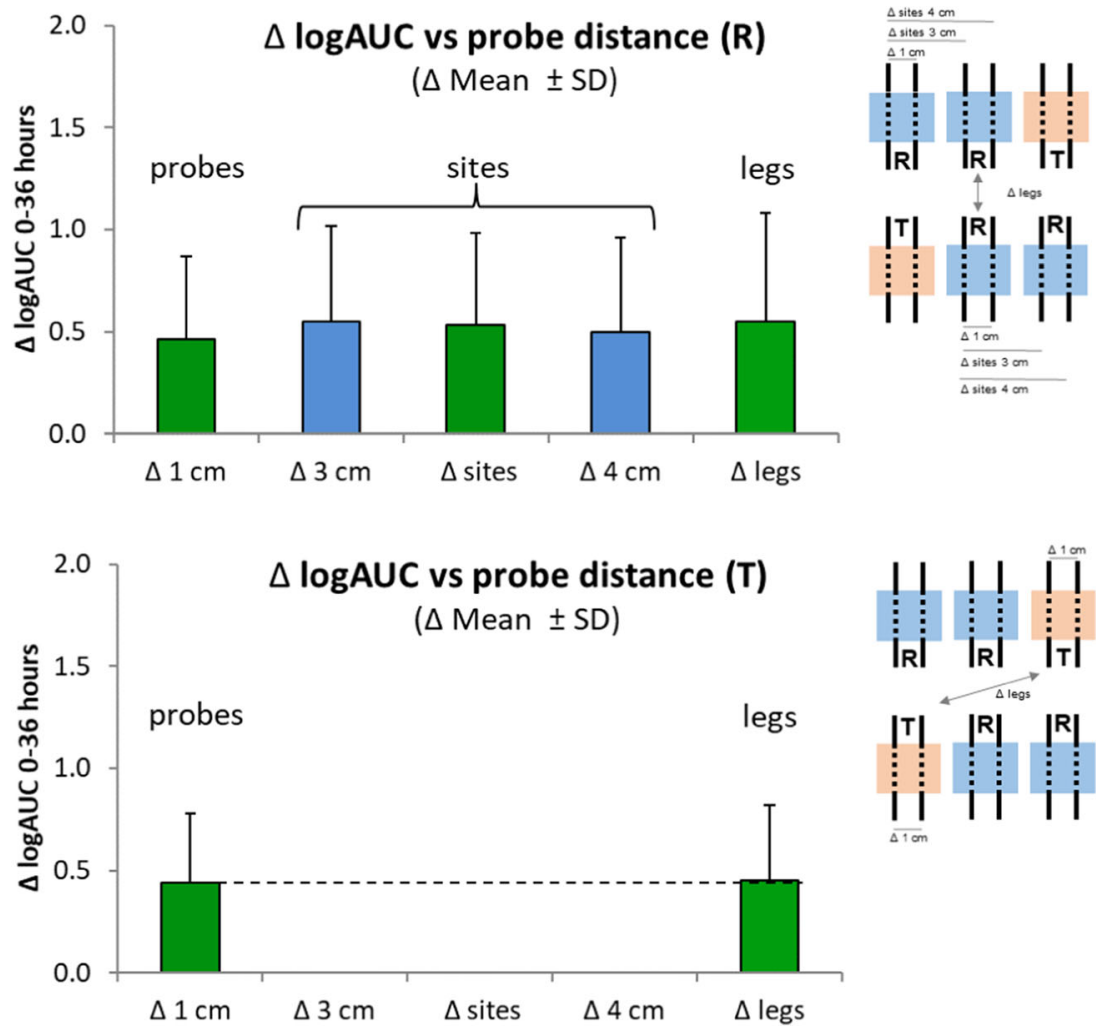

populations of approximately 20 subjects [1 1]. Collectively, our data and the results summarized above may suggest that the intra-subject variability (which is a determinant of the power of a dOFM BE study) may arise, at least in part, from highly localized local 'shunts' in the barrier that have the effect of increasing the variability in dermal PK results. The impact of this variability on a BE study may be minimized by using multiple replicate probes as well as appropriate statistical analyses, using log-transformed data for any statistical comparison. Notably, our data demonstrated that the site selected for a treatment was not a relevant source of variation.

Strengths of our study include a comprehensive data set including data on the dermal PK as well as on biologicalmethodological factors, which allowed the assessment of the sources of variability in dermal PK data after topical drug application and of their impact on topical BE assessments with dOFM. The head-to-head design of the dOFM study allowed for an investigation of intra-subject variability because the study design included positive and negative controls for BE, which had required multiple replicate high-resolution dOFM probes, in particular to enable the positive control, that finally allowed for an identification of the sources of variability.

Nevertheless, our study also has some limitations. We did not analyze the variability of each time point of the acyclovir concentration-time profile but, considering the fact that we wanted to understand the sources of variation in relation to a context of using dOFM to evaluate $\mathrm{BE}$, we focused on the variability of the PK parameter AUC that is routinely utilized as an endpoint for BE assessments. We analyzed AUC, because AUC is assumed to have the highest informational content, being derived from multiple data points describing skin permeation, and we expected that the relative robustness of this parameter might best allow us to identify the sources of variability. Our analysis focussed on the large data set from $\mathrm{R}$ and did not consider potential formulation-specific factors such as skin penetration modifiers, which might exert a local action, modify the recovery, and impact the comparison between $\mathrm{R}$ and $\mathrm{T}$ products. Our expectation was that this risk may be low in future (actual) BE studies for which the $\mathrm{T}$ and $\mathrm{R}$ product components and compositions may be relatively similar. Notably, we used an Austrian acyclovir product as the T product, and it has a different composition than the $\mathrm{R}$ product, which is marketed in the U.S., and which was not expected to be bioequivalent to this $\mathrm{R}$ product. Also, skin conductance and TEWL measurements were not performed directly at the treatment sites. Instead, we analyzed an area of skin close to the treatment site, which might have been slightly different, and thereby, we may have potentially missed some correlation. Finally, our data analysis focused on the variability observed with a single drug (acyclovir), and one that is hydrophilic (unlike most topical drugs which are hydrophobic), and which exhibits very low skin permeation. As a consequence, while our results may be relevant to other hydrophilic drugs with similar penetration properties, it is not evident to what extent these results may also be relevant to relatively faster penetrating, hydrophobic topical drugs that can 
achieve relatively higher dermal PK concentrations. Continuing studies have been initiated to further elaborate on the sources of variability, starting with a moderately lipophilic, fast penetrating drug. Probe-to-probe data variability in the dermis was mainly observed when the drug was applied topically, but not when it was applied systemically, suggesting that our findings from acyclovir may potentially be extrapolated to more lipophilic drugs.

Our findings and conclusions should not be extrapolated to other studies, which are designed for a different purpose and use different equipment under different conditions. Our findings and conclusions, however, can most likely be extrapolated to other clinical studies performed for the purpose of comparative head-to-head topical BE assessments, which use implantable small dermal probes in combination with precision pumps for continuous sampling under highly standardized conditions.

\section{CONCLUSION}

This comprehensive analysis of what is, to date, the largest dermal acyclovir data set obtained by continuous dOFM sampling, has characterized the main sources of variability in a topical dOFM BE study and provided information on the relevance of these sources of variability for topical BE assessments. The results are based upon data from a dOFM study with a hydrophilic topical drug, relatively low amounts of which permeate into the skin. Inter-subject variability dominated the overall variability, and was caused by inter-subject skin barrier differences, but due to the head-to-head study design, the inter-subject variability does not influence the $\mathrm{BE}$ assessment in dOFM studies.

We found a rather low intra-subject variability which is the key for a statistically powerful head-to-head BE study design. None of the methodological factors accounted for this intrasubject variability, and the characteristics of our data support the hypothesis that a significant proportion of the observed intra-subject variability in topical studies might be caused by local skin-related biological factors, e.g. hair follicles, when using a hydrophilic drug with low permeation, like acyclovir.

Additional BE studies would help to further characterize the variability of BE data for topical drugs with different physicochemical properties and/or with greater amounts permeating into the skin. The insights from the work reported here about variability in topical bioavailability and cutaneous pharmacokinetics, and about the source of this variability, directly informs the considerations for the appropriate design/setup of successful BE studies with a minimal number of subjects. The finding that sites do not contribute to variability may mean that any randomization of $\mathrm{T}$ and $\mathrm{R}$ sites is acceptable. The low intra-subject variability supports the concept of a head-tohead BE analysis of topical products ( $\mathrm{T}$ vs $\mathrm{R}$ ) within a relatively small number of subjects that can adequately power statistical conclusions. The inference that the variability of intra-subject data is of a biological origin linked to localized variation in skin microstructures confirms the appropriateness of using replicate probes to characterize the dermal PK of each product. Finally, the disclosure of the data characteristics reported here may prompt the exploration of alternative ways of data analyses. Thus, such a comprehensive data analysis supports the optimization of topical dOFM BE study designs, and the potential development of other efficient methods for topical BE assessments that may promote the availability of safe and effective generic topical products.

\section{ACKNOWLEDGEMENTS}

The authors thank Bernd Tschapeller and Christian Krainer for data management, Sonja Kainz, Peter Reisenegger, Jürgen Lancaj, Joanna Adamczak, and Christian Höfferer (Joanneum Research Forschungsgesellschaft m.b.H) and Stefanie Sach-Friedl, Eva Ekardt, Stefan Korsatko, Gerd Schwagerle, Sarah Bischof, Robert Lipp, and Martina Brunner (Medical University, Graz, Austria) for their help with the clinical study, Denise Kollmann, Stefanie Weiss, and Anton Mautner (Joanneum Research Forschungsgesellschaft m.b.H) for help with bioanalytical sample analysis.

The authors thank Priyanka Ghosh and Tannaz Ramezanli (U.S. Food and Drug Administration, FDA) for scientific collaboration and project administration, and Isadore Kanfer (Rhodes University, Grahamstown, South Africa) and Mike Roberts (University of Queensland, Brisbane, Australia) for scientific discussions. The authors thank Selma Mautner (Joanneum Research, HEALTH Institute for Biomedicine and Health Sciences, Graz, Austria and Medical University of Graz, Division of Endocrinology and Diabetology, Graz, Austria) for critical review and editorial assistance with the manuscript.

Funding for this project was made possible, in part, by the U.S. Food and Drug Administration through research awards 1U01FD004946 and 1U01FD005861. The views expressed in this publication do not reflect the official policies of the FDA, or the Department of Health and Human Services; nor does any mention of trade names, commercial practices, or organization imply endorsement by the U.S. Government.

Open Access This article is licensed under a Creative Commons Attribution 4.0 International License, which permits use, sharing, adaptation, distribution and reproduction in any medium or format, as long as you give appropriate credit to the original author(s) and the source, provide a link to the Creative Commons licence, and indicate if changes were made. The images or other third party material in this article 
are included in the article's Creative Commons licence, unless indicated otherwise in a credit line to the material. If material is not included in the article's Creative Commons licence and your intended use is not permitted by statutory regulation or exceeds the permitted use, you will need to obtain permission directly from the copyright holder. To view a copy of this licence, visit http://creativecommons.org/licenses/by/4.0/.

\section{REFERENCES}

1. Benfeldt E, Hansen SH, Vølund A, Menné T, Shah VP. Bioequivalence of topical formulations in humans: evaluation by dermal microdialysis sampling and the dermatopharmacokinetic method. J Invest Dermatol. 2007 Jan;127(1):170-8.

2. Bodenlenz M, Tiffner KI, Raml R, Augustin T, Dragatin C, Birngruber T, et al. Open flow microperfusion as a dermal pharmacokinetic approach to evaluate topical bioequivalence. Clin Pharmacokinet. 2016;56(1):99.

3. Krishnaiah YSR, Xu X, Rahman Z, Yang Y, Katragadda U, Lionberger R, et al. Development of performance matrix for generic product equivalence of acyclovir topical creams. Int J Pharm [Internet]. 2014;475(1-2):110-22. Available from:. https://doi. org/10.1016/j.ijpharm.2014.07.034.

4. N'Dri-Stempfer B, Navidi WC, Guy RH, Bunge AL. Improved bioequivalence assessment of topical dermatological drug products using dermatopharmacokinetics. Pharm Res. 2009 Feb;26(2):31628.

5. Ng S-F, Rouse JJ, Sanderson FD, Meidan V, Eccleston GM. Validation of a static Franz diffusion cell system for in vitro permeation studies. AAPS PharmSciTech. 2010 Sep;11(3):1432-41.

6. Raney SG, Franz TJ, Lehman PA, Lionberger R, Chen M-L. Pharmacokinetics-based approaches for bioequivalence evaluation of topical dermatological drug products. Clin Pharmacokinet. 2015 Nov 1 1;54(11):1095-106.

7. Kreilgaard M, Kemme MJB, BurggraafJ, Schoemaker RC, Cohen AF. Influence of a microemulsion vehicle on cutaneous bioequivalence of a lipophilic model drug assessed by microdialysis and pharmacodynamics. Pharm Res. 2001;18(5):593-9.

8. Simonsen L, Jørgensen A, Benfeldt E, Groth L. Differentiated in vivo skin penetration of salicylic compounds in hairless rats measured by cutaneous microdialysis. Eur J Pharm Sci. 2004 Feb;2 1(23):379-88.

9. Kreilgaard M. Assessment of cutaneous drug delivery using microdialysis. Adv Drug Deliv Rev. 2002;54(SUPPL.1):S99-121.

10. García Ortiz P, Hansen SH, Shah VP, Sonne J, Benfeldt E. Are marketed topical metronidazole creams bioequivalent? Evaluation by in vivo microdialysis sampling and tape stripping methodology. Skin Pharmacol Physiol. 201 1;24(1):44-53.

11. McCleverty D, Lyons R, Henry B. Microdialysis sampling and the clinical determination of topical dermal bioequivalence. Int $\mathrm{J}$ Pharm. 2006 Feb 3;308(1-2):1-7.

12. Tettey-Amlalo RNO, Kanfer I, Skinner MF, Benfeldt E, Verbeeck RK. Application of dermal microdialysis for the evaluation of bioequivalence of a ketoprofen topical gel. Eur J Pharm Sci. 2009 Feb 15;36(2-3):219-25.

13. Stagni G, O’Donnell D, Liu YJ, Kellogg DL, Morgan T, Shepherd AMM. Intradermal microdialysis: kinetics of iontophoretically delivered propranolol in forearm dermis. J Control Release. 2000;63(3):331-9.
14. Schwingenschuh S, Scharfetter H, Martinsen OG, Boulgaropoulos B, Augustin T, Tiffner KI, et al. Assessment of skin permeability to topically applied drugs by skin impedance and admittance. Physiol Meas. 2017;38(11):N138-50.

15. Pinnagoda J, Tupker RA, Smit JA, Coenraads PJ, Nater JP. The intra- and inter-individual variability and reliability of transepidermal water loss measurements. Contact Dermatitis. 1989;21(4):2559.

16. Møss J. The effect of 3 moisturisers on skin surface hydration. Skin Res Technol. 1996.

17. Southwell D, Barry BW. Penetration enhancement in human skin; effect of 2-pyrrolidone, dimethylformamide and increased hydration on finite dose permeation of aspirin and caffeine. Int J Pharm. 1984;22:291-8.

18. Sjögren F, Anderson C. Sterile trauma to normal human dermis invariably induces IL1beta, IL6 and IL8 in an innate response to "danger". Acta Derm Venereol [Internet]. 2009;89(5):459-65 Available from: http://www.ncbi.nlm.nih.gov/pubmed/ 19734969.

19. Bodenlenz M, Dragatin C, Liebenberger L, Tschapeller B, Boulgaropoulos B, Augustin T, et al. Kinetics of Clobetasol-17propionate in psoriatic Lesional and non-Lesional skin assessed by dermal open flow microperfusion with time and space resolution. Pharm Res [Internet]. 2016;33(9):2229-38 Available from: http:// link.springer.com/10.1007/s1 1095-016-1960-y.

20. Skobe M, Detmar M. Structure, function, and molecular control of the skin lymphatic system. J Investig Dermatol Symp Proc [Internet]. 2000;5(1):14-9. Available from:. https://doi.org/10. 1046/j.1087-0024.2000.00001.x.

21. Khan GM, Frum Y, Sarheed O, Eccleston GM, Meidan VM. Assessment of drug permeability distributions in two different model skins. Int J Pharm. 2005;303(1-2):81-7.

22. Frum Y, Eccleston GM, Meidan VM. Evidence that drug flux across synthetic membranes is described by normally distributed permeability coefficients. Eur J Pharm Biopharm. 2007;67(2): 434-9.

23. Frum Y, Khan GM, SefcikJ, Rouse J, Eccleston GM, Meidan VM. Towards a correlation between drug properties and in vitro transdermal flux variability. Int J Pharm. 2007;336(1):140-7.

24. Meidan VM, Roper CS. Inter- and intra-individual variability in human skin barrier function: a large scale retrospective study. Toxicol Vitr [Internet]. 2008;22(4):1062-9 Available from : http://www.ncbi.nlm.nih.gov/pubmed/18321675.

25. Meidan VM, Pritchard D. A two-layer diffusive model for describing the variability of transdermal drug permeation. Eur J Pharm Biopharm. 2010 Mar;74(3):513-7.

26. Ogiso T, Shiraki T, Okajima K, Tanino T, Iwaki M, Wada T. Transfollicular drug delivery: penetration of drugs through human scalp skin and comparison of penetration between scalp and abdominal skins in vitro. J Drug Target. 2002 Aug;10(5):369-78.

27. Trauer S, Lademann J, Knorr F, Richter H, Liebsch M, Rozycki C, et al. Development of an in vitro modified skin absorption test for the investigation of the follicular penetration pathway of caffeine. Skin Pharmacol Physiol. 2010;23(6):320-7.

28. Schaefer H, Lademann J. The role of follicular penetration: a differential view. Skin Pharmacol Appl Ski Physiol. 2001;14(SUPPL. 1):23-7.

29. Teichmann A, Otberg N, Jacobi U, Sterry W, Lademann J. Follicular penetration: development of a method to block the follicles selectively against the penetration of topically applied substances. Skin Pharmacol Physiol. 2006;19(4):216-23.

30. Patzelt A, Lademann J. Drug delivery to hair follicles. Expert Opin Drug Deliv. 2013;10(6):787-97.

31. Frum Y, Bonner MC, Eccleston GM, Meidan VM. The influence of drug partition coefficient on follicular penetration: in vitro human skin studies. Eur J Pharm Sci [Internet]. 2007;30(3-4):280-7 
Available from: http://www.ncbi.nlm.nih.gov/pubmed/ 17188470

32. Barry BW. Drug delivery routes in skin: a novel approach. Adv Drug Deliv Rev. 2002;54:31-40.

33. Otberg N, Richter H, Schaefer H, Blume-Peytavi U, Sterry W, Lademann J. Variations of hair follicle size and distribution in different body sites. J Invest Dermatol. 2004;122(1):14-9.
34. Xu H, Fonseca M, Wolner Z, Chung E, Wu X, Geller S, et al. Reference values for skin microanatomy: A systematic review and meta-analysis of ex vivo studies. J Am Acad Dermatol. 2017;77(6): 1133-1144.e4.

Publisher's Note Springer Nature remains neutral with regard to jurisdictional claims in published maps and institutional affiliations. 